\title{
INVESTIGAÇÃO NUMÉRICA DA IMPORTÂNCIA DE ARMADURAS DE FRETAGEM EM VIGAS PÓS-TRACIONADAS NÃO ADERENTES
}

\author{
J. P. B. ALMEIDA ${ }^{1}$, R. S. NICOLETTII ${ }^{2}$, A. R. V. WOLENSKI ${ }^{3}$, A. S. C. SOUZA ${ }^{4}$, A. L. CHRISTOFORO ${ }^{5}$ \\ Universidade Federal de São Carlos \\ boff.joaopaulo@gmail.com ${ }^{1}$
}

Submetido 18/02/2020 - Aceito 08/04/2020

DOI: $10.15628 /$ holos.2020.9566

\section{RESUMO}

No concreto protendido, em razão da aplicação da força de protensão, a região das ancoragens é submetida a elevadas tensões. Ao longo de um trecho denominado comprimento de regularização, a distribuição dessas tensões não é uniforme, ocorrendo tensões nas direções transversais do elemento. Para resistir aos esforços transversais de tração, faz-se necessária a adoção de armaduras complementares, denominadas armaduras de fretagem. Nesse contexto, este trabalho teve como objetivo investigar o comportamento e a distribuição de tensões ao longo do comprimento de regularização de elementos protendidos, avaliando numericamente, no software Abaqus ${ }^{\circledR}$, a influência e importância das armaduras de fretagem em tais elementos. Para tanto, foram simulados quatro modelos numéricos, a saber, VE,
\end{abstract}

VEF-3, VEF-4 e VEF-5, com mesma quantidade de armadura longitudinal e transversal, diferenciando-se pelo fato de os modelos VEF-3, VEF-4 e VEF-5 possuírem, respectivamente, 3,4 e 5 estribos adicionais de fretagem em cada uma das extremidades da viga. Verificou-se que a inserção dos estribos adicionais não promoveu alterações significativas na falha à compressão na região das ancoragens, todavia promoveu reduções relevantes na falha à tração. Além disso, no modelo VE, o valor da tensão na armadura atingiu a tensão de escoamento do aço, enquanto nos modelos VEF-3, VEF-4 e VEF-5 as tensões reduziram $19,48 \%, 56,58 \%$ e $57,96 \%$ em relação ao VE. Tal fato evidencia a importância de armaduras de fretagem em atendimento à segurança de vigas póstracionadas sem aderência.

PALAVRAS-CHAVE: Concreto protendido, pós-tração, comprimento de regularização, análise numérica.

\section{INVESTIGATION OF THE IMPORTANCE REINFORCEMENT IN THE ANCHORAGE ZONE OF UNBONDED POST-TENSIONED CONCRETE BEAMS}

\begin{abstract}
In prestressed concrete, due to the application of the prestressing force, the anchorages zones are subjected to high stresses. Along a stretch, called anchorage zone, the distribution of these stresses is not uniform, with transversal stress acting in the structural element. To resist the transverse stresses, it is necessary to adopt complementary reinforcement, called anchorage zone reinforcement. In this context, this paper aimed to investigate the behavior and distribution of stresses along the regularization length of prestressed elements, numerically evaluating in the Abaqus ${ }^{\circledR}$ software the influence and importance of anchorage zone reinforcement on such elements. For this purpose, four numerical models were simulated, VE, VEF-3, VEF-4 and
\end{abstract}

VEF-5, with the same area of longitudinal and transversal reinforcement, differing in the fact that the VEF-3, VEF-4 and VEF- 5 have, respectively, 3, 4 and 5 additional stirrups at each end of the beam. It was found that the insertion of the additional stirrups did not promote significant changes in the compression failure in the anchorage region, however it did promote relevant reductions in the failure in the traction. In addition, in the VE model, the value of the stress in the armature reached the yield strength of the steel, while in the models VEF-3, VEF- 4 and VEF-5 the stresses reduced $19.48 \%, 56.58 \%$ and $57.96 \%$ in relation to VE. This fact highlights the importance of anchorage zone reinforcement to assegurate the safety of unbonded post-tensioned beams.

KEYWORDS: Prestressed concrete, Post-tensioning, anchorage zone, numerical analysis. 


\section{INTRODUÇÃO}

$\mathrm{Na}$ construção civil, um dos grandes desafios encontrados é conciliar as necessidades arquitetônicas com métodos construtivos eficazes. Diante disso, o concreto protendido apresentase como solução adequada. Em relação ao concreto armado, o concreto protendido apresenta várias vantagens: as deformações provenientes das cargas de serviço são melhor controladas e as fissuras no concreto são praticamente eliminadas, devido às tensões de compressão induzidas pela força de protensão.

Economicamente, a combinação da elevada tensão da armadura ativa com a alta tensão de compressão do concreto conduz ao melhor aproveitamento de ambos os materiais, permitindo seções transversais menores. Por essas e várias outras vantagens, nas últimas décadas, o concreto protendido tornou-se amplamente utilizado, tanto em lajes e vigas com grandes vãos como em obras de pontes (YUN, 2005).

Diversos autores têm se dedicado ao estudo do comportamento das estruturas em concreto protendido, corroborando seus diversos benefícios, bem como validando e desenvolvendo procedimentos de cálculo (DE OLIVEIRA e JÚNIOR, 2016; MARTí et al., 2016; MOURA et al., 2018). No mais, os trabalhos recentes dividem-se basicamente no estudo de peças de concreto protendido com armadura não aderente (DU et al., 2016; MOREIRA et al., 2018; BONOPERA et al., 2019) e elementos de concreto protendido reforçados com fibras (CHEN et al., 2015; SHIN et al., 2016; BISCHOFF et al., 2018).

Todavia, o fato de a protensão introduzir ações na estrutura exige do engenheiro uma atenção especial no dimensionamento de elementos protendidos. Verificações como, por exemplo, estado limite de descompressão (ELS-D), estado limite de compressão excessiva (ELS-CE) e estado limite último no ato da protensão (ELU-ATO), não necessárias no dimensionamento de elementos em concreto armado, devem ser atendidas no dimensionamento em concreto protendido. Portanto, o dimensionamento de peças protendidas apresenta particularidades. Dentre tais, tem-se o dimensionamento das armaduras de fretagem.

As armaduras de fretagem, utilizadas em forma de espirais, estribos secundários, ou a combinação de ambos, são necessárias para resistir às tensões de tração transversais existentes no elemento estrutural devido à aplicação da força de protensão. Essas tensões podem causar o fendilhamento longitudinal no elemento protendido, necessitando, portanto, da utilização de armaduras de reforço da zona de ancoragem (HAROON et al., 2006).

Estudos acerca da armadura de fretagem e de seus efeitos na estrutura têm sido conduzidos por diversos pesquisadores na literatura internacional, tanto no desenvolvimento de sistemas de ancoragem quanto no estudo do comportamento e distribuição de tensões no elemento estrutural. Inicialmente, destacam-se os realizados por Mörsh (1924), os quais apresentaram um modelo baseado no equilíbrio de forças para visualizar a distribuição de tensões em peças estruturais carregadas com forças concentradas.

Desde então, um grande número de pesquisas sobre zonas de ancoragem foi produzido utilizando a teoria da elasticidade, o método dos elementos finitos e alguns estudos experimentais 
(KOMENDANT, 1952; GUYON, 1953; ZIELINSKI e ROWE, 1960; BASE et al., 1966; GERGELY e SOZEN, 1967; YETTRAM e ROBBINS, 1969; YUN, 2005; HAROON et al., 2006; AXSON, 2008; BONETTI et al., 2014; CERVENKA e GANZ, 2014; HOU et al., 2017; KWON et al., 2017; KIM et al., 2018; MAO et al., 2018).

Tais estudos colaboraram para o entendimento dos estados de tensão de elementos protendidos, contribuindo para revisões de documentos normativos internacionais. Todavia, no Brasil, o documento normativo ABNT NBR 6118 (2014) intitulado "Projeto de estruturas de concreto - Procedimento" não faz menção aos procedimentos para dimensionamento de armaduras de fretagem, o que motiva o desenvolvimento de trabalhos nesta temática, expondo os estudos bibliográficos acerca do assunto e expondo resultados que contribuam para a revisão do documento normativo brasileiro.

Diante do exposto, este trabalho teve como objetivos: apresentar informações por meio de revisões bibliográficas acerca do estado de tensão ao longo do comprimento de regularização de elementos protendidos; sintetizar o roteiro de cálculo para o dimensionamento de armaduras de fretagem em vigas pós-tracionadas sem aderência, tomando-se como referência os códigos normativos nacionais e internacionais; e avaliar numericamente a influência e importância de armaduras de fretagem em tais elementos estruturais, pautando-se no fato de que as normas brasileiras não abordam esse assunto.

\section{FUNDAMENTAÇÃO TEÓRICA}

Para compreender o comportamento das armaduras de fretagem, é de vital importância entender a distribuição de tensões nas extremidades de vigas protendidas. Com esse intuito, apresenta-se o comportamento básico de blocos parcialmente carregados e os modos de falha da zona de regularização de vigas pós-tracionadas.

\subsection{Blocos parcialmente carregados}

Vigas protendidas pós-tracionadas são submetidas a uma alta concentração de tensões de compressão em sua extremidade, devido à transferência da força de protensão por meio de ancoragens mecânicas (HAROON et al., 2006).

De acordo com Fusco (2013), as ancoragens dos cabos de protensão posicionadas no centro de gravidade da seção transversal da peça de concreto possuem o funcionamento básico de um bloco parcialmente carregado, no qual, ao longo de um certo trecho, denominado comprimento de regularização $\left(\ell_{0}\right)$, a distribuição de tensões não é uniforme, estando a tensão longitudinal de compressão acompanhada por tensões de tração nas direções transversais (vide Figura 1).

Pelo princípio de Saint Venant, o comprimento de regularização é na ordem de grandeza da maior dimensão "a" da seção transversal da peça (IYENGAR e YOGANANDA, 1966; ZHI-QI e ZHAO, 2011; ZHAO et al., 2011). 


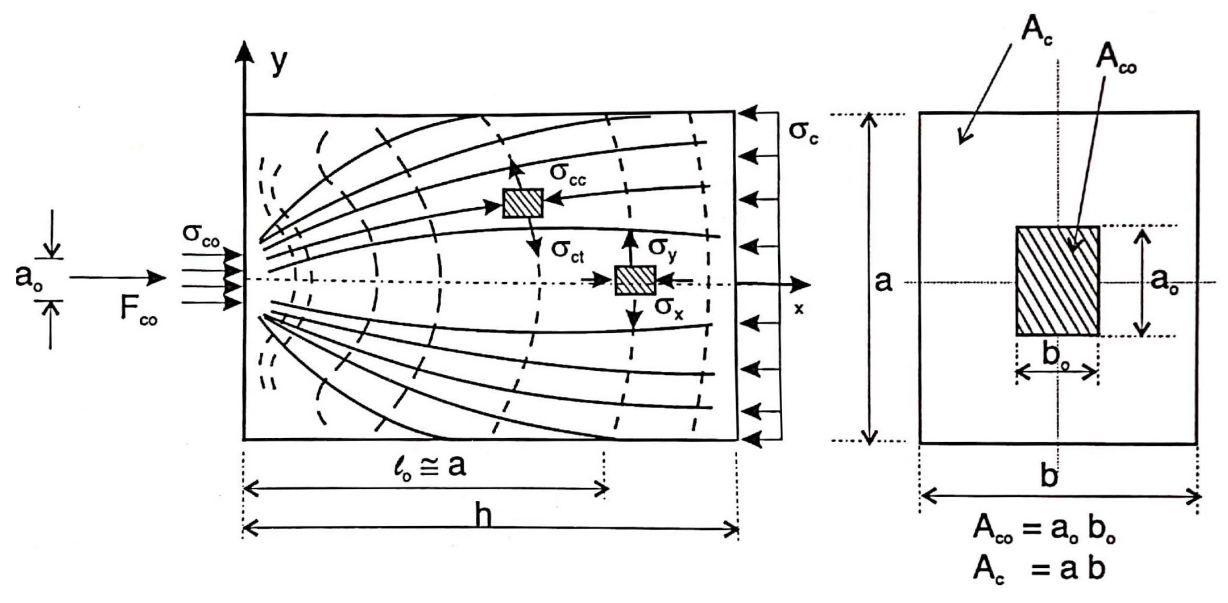

Figura 1: Distribuição de tensões em blocos parcialmente carregados.

Fonte: Fusco (2013).

A Equação (1) descreve a intensidade da tensão de compressão $\sigma_{c 0}$ atuante na direção longitudinal da peça gerada pela aplicação da força de $F_{c 0}$ na área $A_{c 0}$.

$$
\sigma_{c 0}=\frac{F_{c 0}}{A_{c 0}}=\frac{F_{c 0}}{a_{0} \cdot b_{0}}
$$

Pelo fato de a força $F_{c 0}$ ser aplicada em uma área parcial, o bloco fica sujeito a estados múltiplos de tensão. Nessas condições, na direção longitudinal, a tensão $\sigma_{x}$ será sempre de compressão, enquanto, nas direções transversais, as tensões $\sigma_{y}$ e $\sigma_{z}$ serão de compressão apenas na região próxima à face de aplicação da força $F_{c 0}$ e de tração no trecho remanescente do comprimento de regularização, conforme demonstra a Figura 2 (YETTRAM e ROBBINS, 1969; HAROON et al., 2006; ZHI-QI e ZHAO, 2011; FUSCO, 2013).

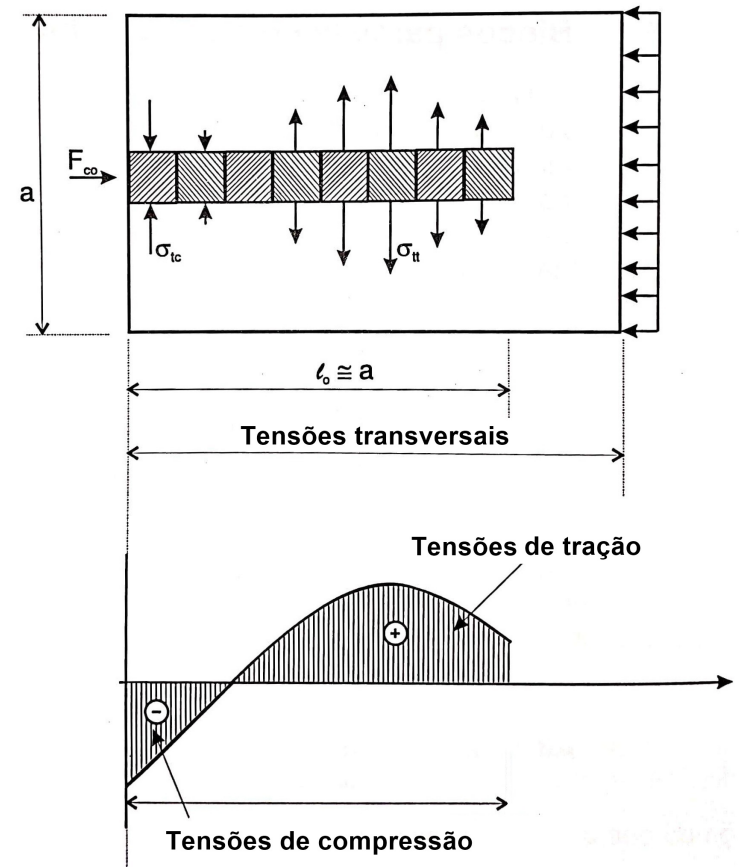

Figura 2: Distribuição das tensões transversais em blocos parcialmente carregados. Fonte: Adaptado de Fusco (2013). 
Como guia geral para o estudo das zonas de regularização, a ABNT NBR 6118 (2014) prescreve que a difusão da força de protensão pode ser considerada a partir do dispositivo de ancoragem, por meio de um ângulo limitado por duas retas inclinadas orientadas a $\beta$ graus em relação à direção de aplicação da força de protensão, sendo tan $\beta=2 / 3$ (Figura 3). Tal regra é empregada para a localização das seções onde as tensões já estão totalmente regularizadas, ou seja, a tensão de compressão na direção longitudinal é uniforme ao longo de toda a seção transversal, não havendo tensões nas direções transversais.

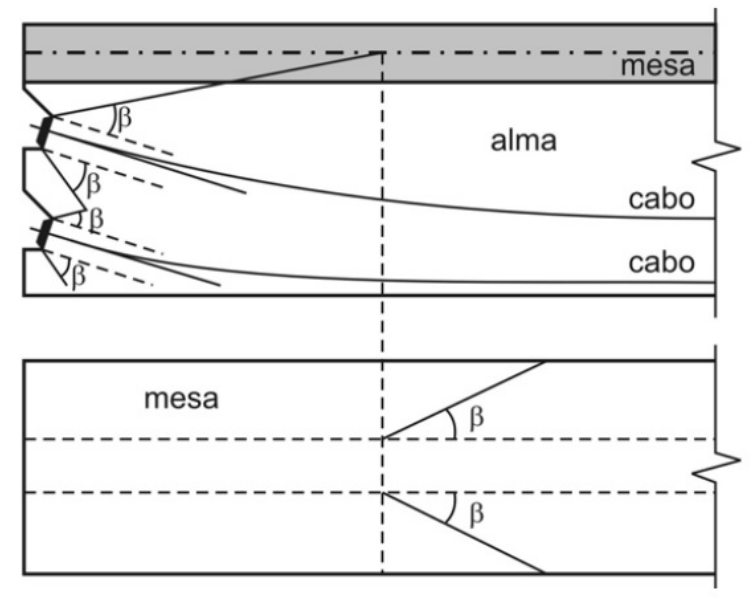

Figura 3: Zona de regularização na alma e na mesa. Fonte: ABNT NBR 6118 (2014).

2.2 Procedimentos de cálculo da literatura

\subsubsection{Mörsh (1924)}

Mörsh (1924), com o intuito de descrever o efeito da força de ruptura ao longo do comprimento de regularização, apresentou um modelo fundamentado no fluxo de tensões em um elemento carregado de forma concêntrica (Figura 4). Tal modelo, conhecido atualmente como método das bielas e tirantes, esquematiza a difusão das tensões axiais de compressão como uma estrutura equivalente em equilíbrio, constituída por elementos comprimidos (bielas) e tracionados (tirantes) conectados por nós. O método desenvolvido por Mörsh mostrou-se condizente com a distribuição de tensões de acordo com o princípio de Saint Venant (HENGPRATHANEE, 2004). 


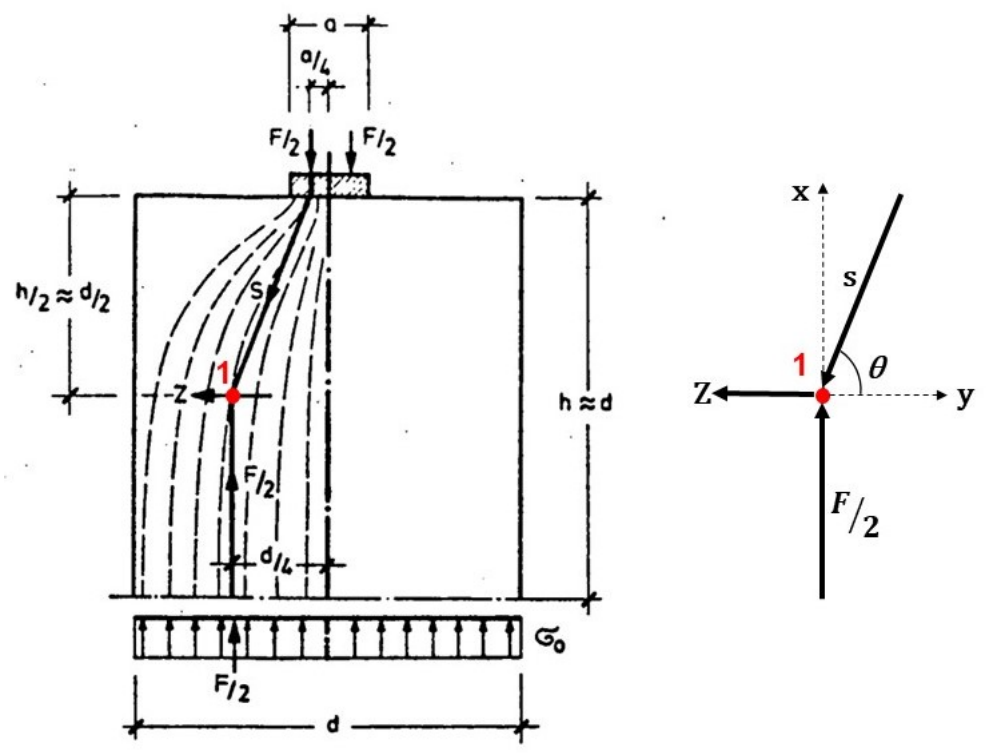

Figura 4: Modelo idealizado de distribuição de tensões em blocos parcialmente carregados segundo Mörsh. Fonte: Adaptado de Burdet (1990)

Em decorrência da difusão das tensões axiais de compressão na direção longitudinal $(x)$ do elemento, tem-se a ocorrência de bielas inclinadas. $O$ equilíbrio dessas bielas se dá com o aparecimento de esforços transversais de tração (Z), atuante nas direções transversais (y e z) que tendem a produzir o fendilhamento longitudinal do elemento estrutural (FUSCO, 2013).

Partindo da consideração de que os esforços máximos transversais de tração (Z) ocorrem a uma distância correspondente à metade do comprimento de regularização $(h / 2 \approx d / 2)$ e impondo o equilíbrio do nó 1 (Figura 4), Mörsh (1924) determinou analiticamente a intensidade do máximo esforço transversal de tração (Z). As Equações (2) a (7) descrevem o procedimento citado.

$$
\begin{aligned}
& \tan (\theta)=\frac{d}{2} \cdot \frac{1}{\left(\frac{d}{4}-\frac{a}{4}\right)}=\frac{2 d}{d-a} \\
& \left(\rightarrow_{+}\right) \sum F_{x}=0:-Z-s \cdot \cos (\theta)=0 \Rightarrow s=\frac{Z}{\cos (\theta)} \\
& \left(\uparrow_{+}\right) \sum F_{y}=0:+\frac{P}{2}-s \cdot \operatorname{sen}(\theta)=0
\end{aligned}
$$

Substituindo a Equação (3) na Equação (4), tem-se:

$$
+\frac{P}{2}-\frac{Z}{\cos (\theta)} \cdot \operatorname{sen}(\theta)=0 \Rightarrow-\frac{P}{2}-Z \cdot \tan (\theta)=0
$$

Substituindo a Equação (2) na Equação (5), tem-se: 


$$
+\frac{P}{2}-Z \cdot \frac{2 d}{d-a}=0 \Rightarrow Z=\frac{P}{2} \cdot \frac{d-a}{2 d}
$$

Simplificando a Equação (6), resulta a Equação (7), que descreve o máximo esforço de tração na direção transversal y em função da força de ruptura $(P)$ e da relação entre as larguras da placa de ancoragem (a) e do elemento estrutural (d).

$$
Z=0,25 \cdot P \cdot\left(1-\frac{a}{d}\right)
$$

A determinação do máximo esforço de tração, atuante na direção transversal z, é realizada de modo análogo, considerando, para tanto, as outras dimensões da placa de ancoragem e do elemento estrutural.

\subsubsection{Guyon (1953)}

Guyon (1953) estudou o comportamento de uma carga concêntrica aplicada sobre um corpo retangular. Os valores de tensão de tração transversais $\left(\sigma_{\mathrm{tt}}\right)$ foram condicionados à intensidade da força $\left(F_{c 0}\right)$ aplicada e à relação entre a largura da placa de ancoragem $\left(a_{0}\right)$ e a largura da seção transversal do bloco parcialmente carregado (a) (HENGPRATHANEE, 2004). A Figura 5 expõe o valor da resultante de transversal de tração $R_{\mathrm{tt}}$, assim como as posições onde ocorrem as tensões mínimas $\left(\sigma_{\mathrm{tt}}=0\right)$ e máximas $\left(\sigma_{\mathrm{tt}, \text { máx }}\right)$.

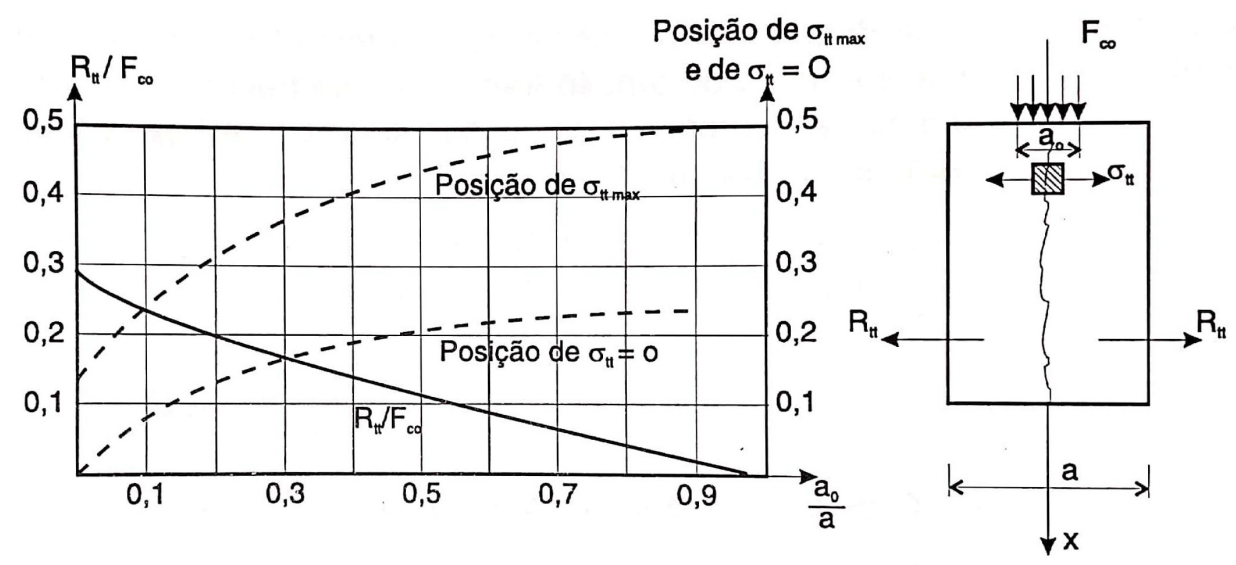

Figura 5: Valor da resultante transversal de tração $\mathbf{R}_{\mathrm{tt}}$. Fonte: Fusco (2013).

De acordo com os resultados apresentados por Guyon (1953), ilustrados pela Figura 6, a força transversal $\left(R_{t t}\right)$ que tende a provocar o fendilhamento longitudinal no bloco é expressa pela Equação (8).

$$
R_{t t}=0,30 \cdot F_{c 0} \cdot\left(1-\frac{a_{0}}{a}\right)
$$

Ressalta-se, com base na Figura 6 e na Equação (8), que, quando a relação ao/a é igual a 1, ou seja, a largura da placa de ancoragem se iguala à largura do bloco, a força transversal de tração 
é nula $\left(R_{t t}=0\right)$. Por sua vez, quando a relação ao/a tende a zero (força pontual), tem-se a intensidade máxima do esforço transversal de tração $\left(R_{\mathrm{tt}}=0,30 \cdot \mathrm{F}_{\mathrm{co}}\right)$, valor esse muito próximo ao determinado por Mörsh (1924) por meio da Equação (7) $(Z=0,25 \cdot P)$.

\subsubsection{ACl 318-19}

A normativa 318-19 do American Concrete Institute ( $\mathrm{ACl}$ ), intitulada "Building Code Requirements for Structural Concrete and Commentary", permite, para a determinação do esforço de tração nas direções transverais, a adoção do método das bielas e tirantes, do método dos elementos finitos e, também, o uso de equações simplificadas, com a ressalva de que as últimas não devem ser empregadas quando o elemento estrutural não apresentar seção transversal retangular. Todavia, em muitos casos, permite-se usar a Equação (9), apresentada pela normativa americana para a estimativa da força de tração transversal ( $\left.T_{\text {burst }}\right)$.

$$
T_{\text {burst }}=0,25 \cdot \sum P_{p u} \cdot\left(1-\frac{h_{a n c}}{h}\right)
$$

Na Equação (9), $\Sigma P_{p u}$ corresponde ao somatório da força máxima de protensão aplicada em cada cabo, enquanto $h_{\text {anc }}$ e $h$ denotam, respectivamente, a dimensão da placa de ancoragem e a do elemento estrutural, na direção considerada. Cabe destacar que a Equação (9), apresentada pela ACl 318-19, é análoga à Equação (7) deduzida por Mörsh (1924), utilizando o método das bielas e tirantes.

\section{METODOLOGIA}

Com o intuito de avaliar a influência das armaduras de fretagem em vigas protendidas, foram desenvolvidos quatro modelos numéricos no software Abaqus ${ }^{\circledR}$, a saber, VE (viga com estribos verticais espaçados uniformemente a cada $17 \mathrm{~cm}$ ), VEF-3 (viga VE com 3 estribos adicionais nas seções de ancoragem), VEF-4 (viga VE com 4 estribos adicionais nas seções de ancoragem) e VEF-5 (viga VE com 5 estribos adicionais nas seções de ancoragem). Mais especificamente, os modelos consistem em vigas com protensão não aderente, biapoiada, com comprimento de vão de $10,40 \mathrm{~m}$, diferenciando-se pelo fato de o modelo VE possuir apenas estribos verticais (diâmetro de 6,3 $\mathrm{mm}$ ) espaçados uniformemente a cada $17 \mathrm{~cm}$, ao longo da viga; enquanto os modelos VEF-3, VEF-4 e VEF-5 possuem, além dos distribuídos uniformemente, 3, 4 e 5 estribos adicionais de fretagem, posicionados em cada extremidade da viga. Desse modo, os modelos VEF-3, VEF-4 e VEF-5 detêm uma quantidade adicional total de 6, 8 e 10 estribos na viga.

Para cada modelo, foram verificados os respectivos danos à compressão e à tração, assim como os valores da tensão von Mises nos estribos. Vale ressaltar que o dano à compressão referese ao trecho descendente do modelo constitutivo do concreto à compressão, no qual o material está sendo esmagado e, em consequência, perde sua resistência e rigidez. Analogamente, o dano à tração também trata-se do ramo descendente do modelo constitutivo do concreto à tração, em que o material está sendo fissurado. Tais danos são irreversíveis e mensurados de forma adimensional, com intensidade variando de 0 a 1, em que 0 representa um material sem dano e 1 , um material totalmente danificado, isto é, praticamente sem qualquer resistência ou rigidez. 
A Figura 6 apresenta o detalhamento transversal das vigas simuladas.

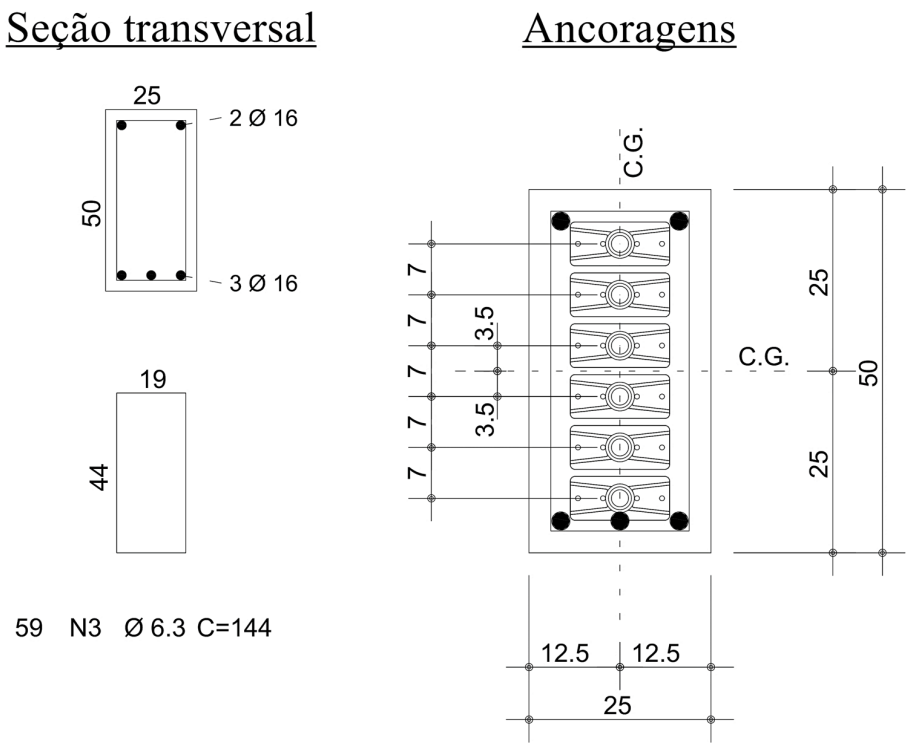

Figura 6: Detalhamento transversal da viga.

Cabe destacar que, no modelo VE, os estribos foram dimensionados, conforme o modelo de cálculo I da ABNT NBR 6118 (2014), para resistir somente aos esforços cortantes provenientes do carregamento atuante. No modelo VEF-4, por sua vez, os estribos adicionais foram dimensionados, com o auxílio da Equação (7), para resistir aos esforços transversais de tração atuantes em razão da aplicação da força de protensão. Por fim, os modelos VEF-3 e VEF-5 foram concebidos com o intuito de analisar a distribuição de tensões com uma quantidade inferior e superior à armadura de fretagem de referência calculada conforme Equação (7).

Ademais, os modelos foram analisados no ato da protensão, instante em que atua a máxima força de protensão e, consequentemente, a maior intensidade das tensões transversais de tração.

\subsection{Elementos e malha}

A viga de concreto foi modelada com elementos sólidos C3D8R da biblioteca do Abaqus $₫$, os quais possuem 8 nós com 3 graus de liberdades por nó (translações nas direções $x, y$ e $z$ ), pois tais elementos suportam a análise plástica com elevadas deformações e deslocamentos, além de ser possível inserir barras de armadura em seu interior. Por sua vez, para as armaduras transversais e longitudinais ativas e passivas, utilizaram-se elementos de treliça T3D2, que possuem 2 nós com 3 graus de liberdades por nó (translações nas direções $x$, y e z) e constituem um eficiente elemento para representar o comportamento de barras, treliças e cabos submetidos a forças axiais. Os elementos de aço e concreto foram vinculados por meio da ferramenta embedded constrain do Abaqus ${ }^{\circledR}$, fazendo com que os graus de liberdade dos nós da armadura fossem restringidos à translação. 
Tabela 1: Tipo dos elementos e respectivas malhas das partes.

\begin{tabular}{lcc}
\hline \multicolumn{1}{c}{ Parte } & Tipo & $\begin{array}{c}\text { Tamanho do elemento } \\
(\mathbf{x}, \mathbf{y}, \mathbf{z})[\mathbf{m m}]\end{array}$ \\
\hline Viga de concreto & C3D8R & $(25,25,25)$ \\
Armadura longitudinal ativa & T3D2 & $(0,0,25)$ \\
Armadura longitudinal passiva & T3D2 & $(0,0,25)$ \\
Armadura transversal - estribos & T3D2 & $(25,25,0)$ \\
\hline
\end{tabular}

(a) Geometria da armadura ativa

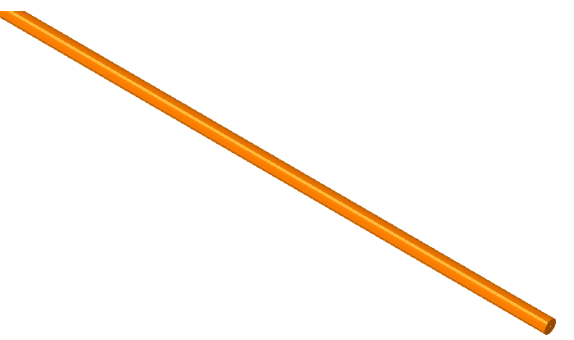

(c) Geometria da armadura passiva

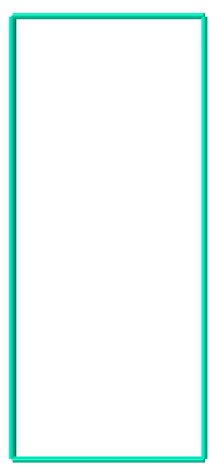

(e) Geometria dos estribos

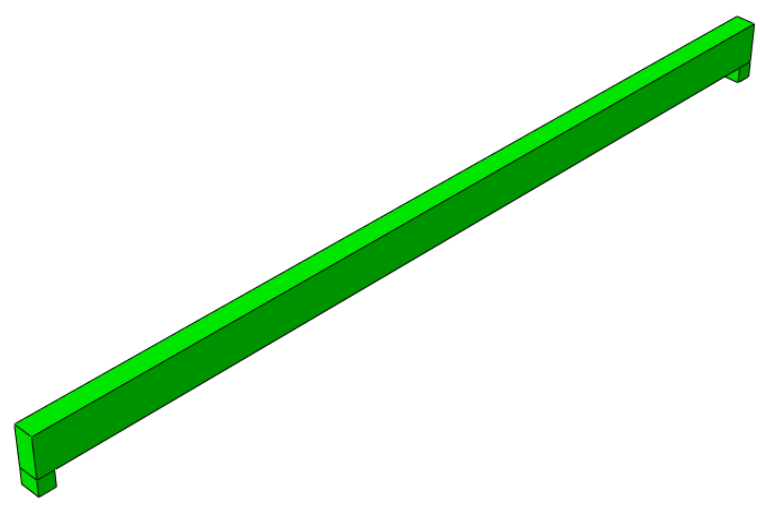

(g) Geometria da viga de concreto (b) Malha da armadura ativa

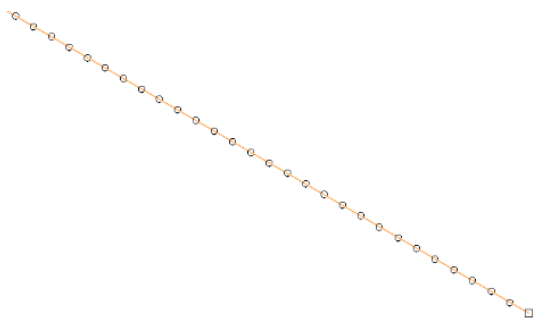

(d) Malha da armadura passiva

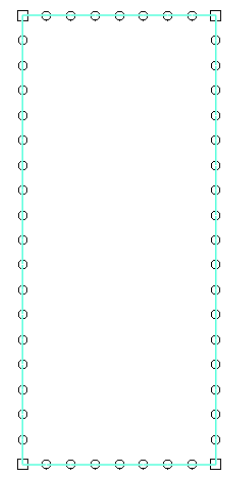

(f) Malha dos estribos

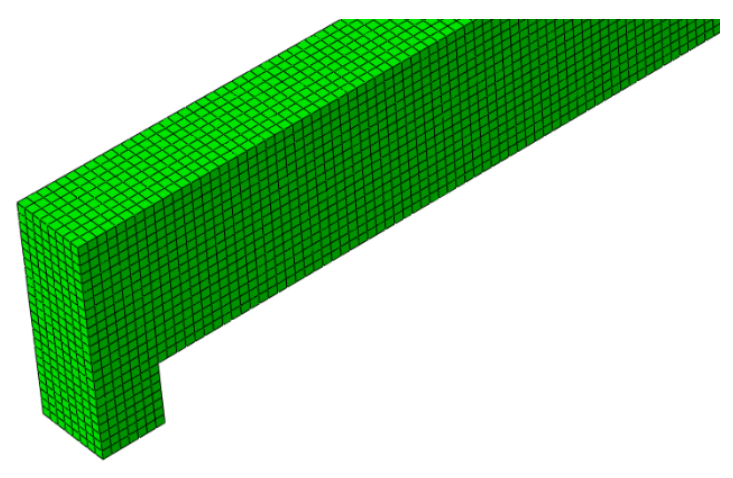

(h) Malha da viga de concreto

Figura 7: Geometria e malha das partes dos modelos. 


\subsection{Propriedades dos materiais}

Quanto às propriedades mecânicas dos materiais, as armaduras passivas e ativas foram descritas por uma relação constitutiva tensão-deformação com comportamento elastoplástico perfeito. Para o concreto, adotou-se o modelo constitutivo Concrete Damaged Plasticity (CDP) de Guo (2014), pois este modelo prevê com grande precisão o comportamento de concretos e outros materiais frágeis como rocha e argamassa, além de considerar os efeitos da fissuração.

Quanto às propriedades dos materiais utilizados na modelagem, tem-se que:

- a tensão de escoamento das armaduras passivas foi $f_{y}=500 \mathrm{Mpa}$ (Aço CA-50), enquanto a das armaduras ativas foi $\mathrm{f}_{\mathrm{y}}=1.700 \mathrm{Mpa}$ (Aço CP 190-RB). O módulo de elasticidade longitudinal de tais aços e o coeficiente de Poisson foram, respectivamente, $\mathrm{E}=200 \mathrm{GPa}$ e $\mathrm{U}=0,3$;

- a resistência à compressão do concreto foi $\mathrm{f}_{\mathrm{c}}=21,0 \mathrm{MPa}$ (resistência mínima exigida no ato da protensão). O módulo de elasticidade longitudinal do concreto e o coeficiente de Poisson foram, respectivamente, $\mathrm{E}=27.485 \mathrm{MPa}$ e $\mathrm{u}=0,2$.

\subsection{Vinculação e carregamento}

As vigas foram consideradas biapoiadas, de modo que o movimento nas direções vertical e longitudinal foi restringido por meio de apoios com a largura da viga, $200 \mathrm{~mm}$ de comprimento e $200 \mathrm{~mm}$ de altura. Os carregamentos, por sua vez, tratam-se do peso próprio da viga (único carregamento solicitante atuante no ato da protensão) e de uma força de protensão de $900 \mathrm{kN}$ (150 kN por cordoalha - considerando-se um coeficiente de ponderação da força de protensão $\gamma_{p}$ igual a 1,1) aplicada ao elemento estrutural. Eles foram aplicados com passos de carga máximos de 1,0\% da carga total. A Figura 8 apresenta o carregamento e as condições de contorno dos modelos.

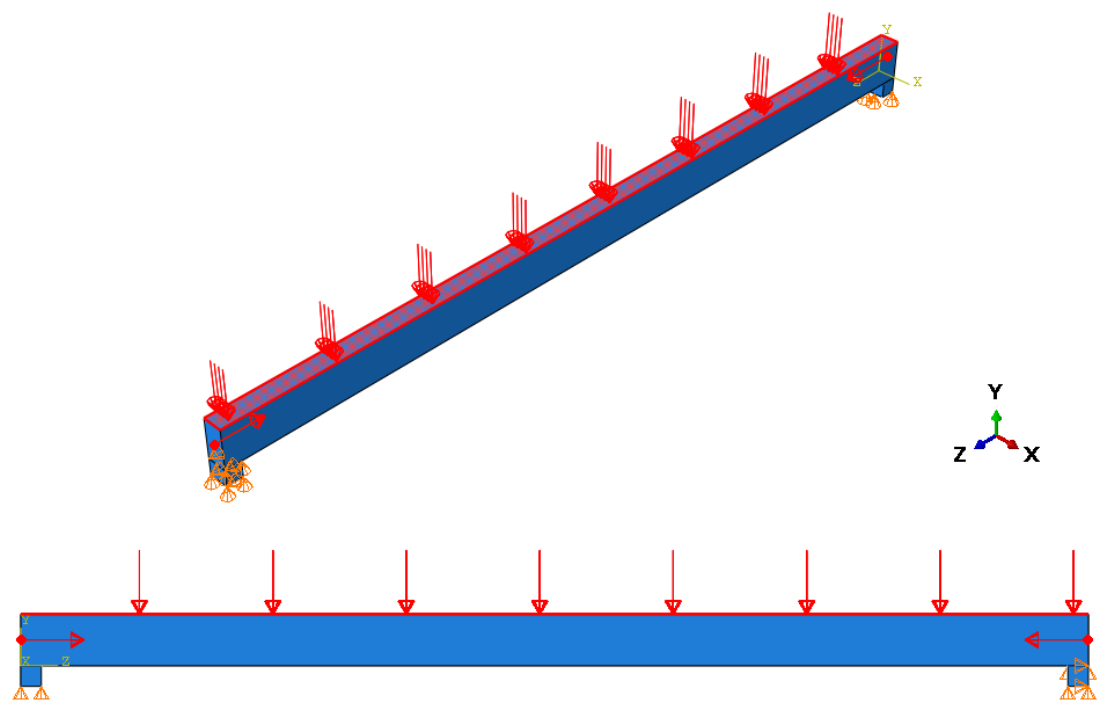

Figura 8: Vinculação e carregamento dos modelos numéricos. 
No mais, todos os modelos foram simulados com a consideração da não linearidade geométrica para considerar os efeitos de segunda ordem.

\subsection{Validação dos modelos numéricos}

Os modelos numéricos foram validados com base no critério da deflexão vertical, analisando-se esta no meio do vão longitudinal para o modelo sem armadura de fretagem.

Para as condições descritas anteriormente, no modelo sem armadura de fretagem, os valores de deflexão analítica e numérica foram de, respectivamente, -0,1861 mm e -0,1785 mm, apresentando um erro relativo de 4,08 \%, calculado conforme Equação 10.

$$
\text { Erro relativo }=\frac{\mid \text { Medida numérica }- \text { Medida analítica } \mid}{\mid \text { Medida analítica } \mid} \cdot 100 \%
$$

Desse modo, considera-se que o modelo numérico está validado.

\section{RESULTADOS E DISCUSSÕES}

As Figuras 9 e 10 apresentam, respectivamente, os danos à compressão e à tração para cada um dos módulos numéricos investigados.

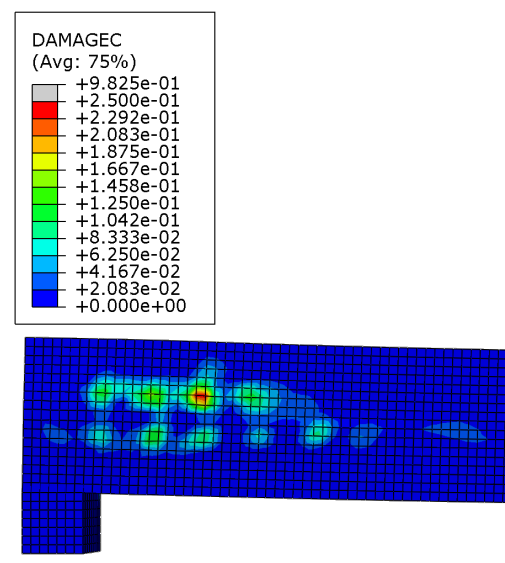

(a)

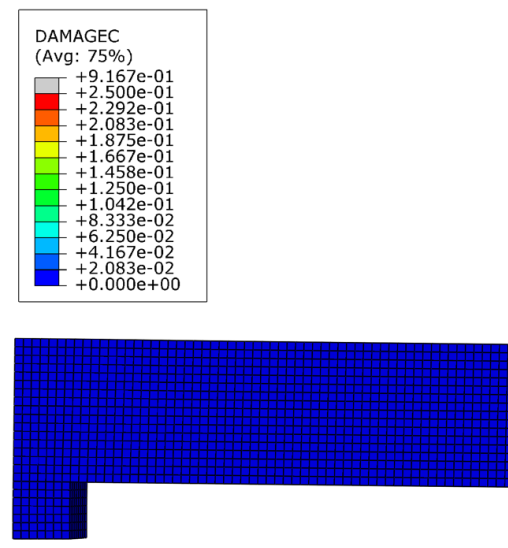

(c)

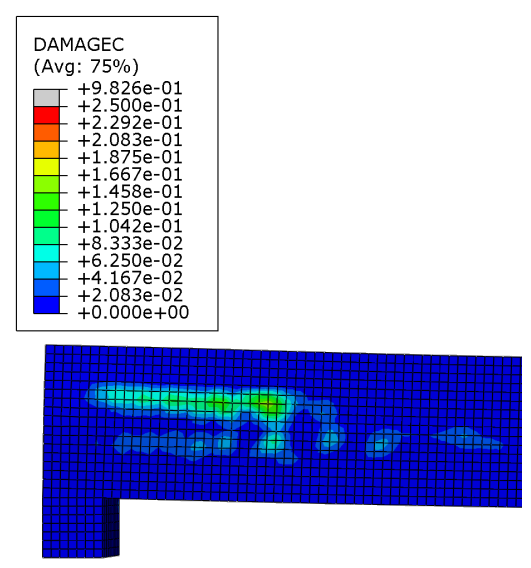

(b)

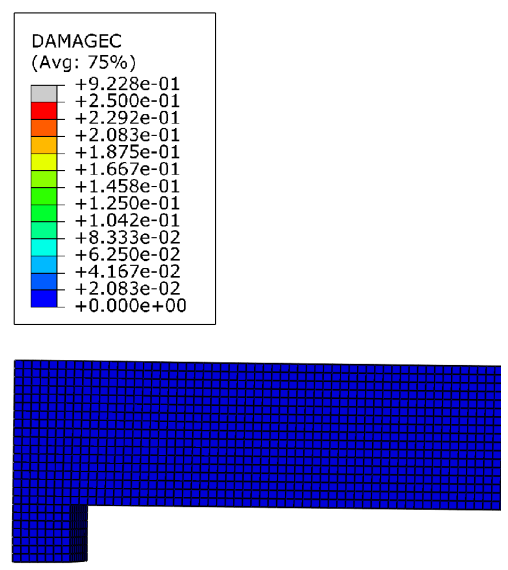

(d)

Figura 9: Dano por compressão: modelo VE (a); modelo VEF-3 (b); modelo VEF-4 (c); modelo VEF-5 (d). 

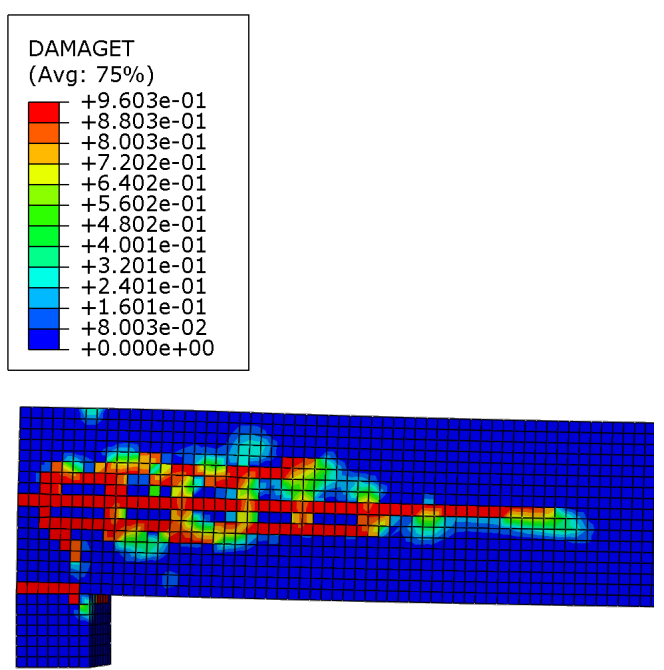

(a)

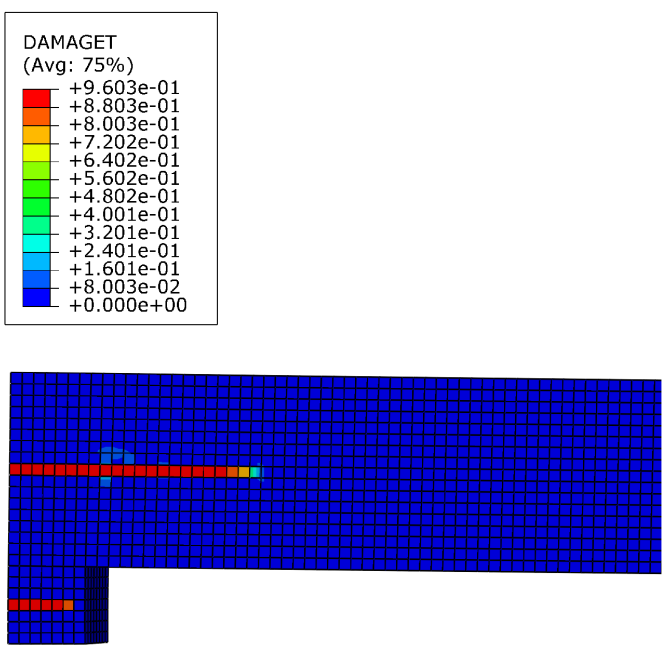

(c)
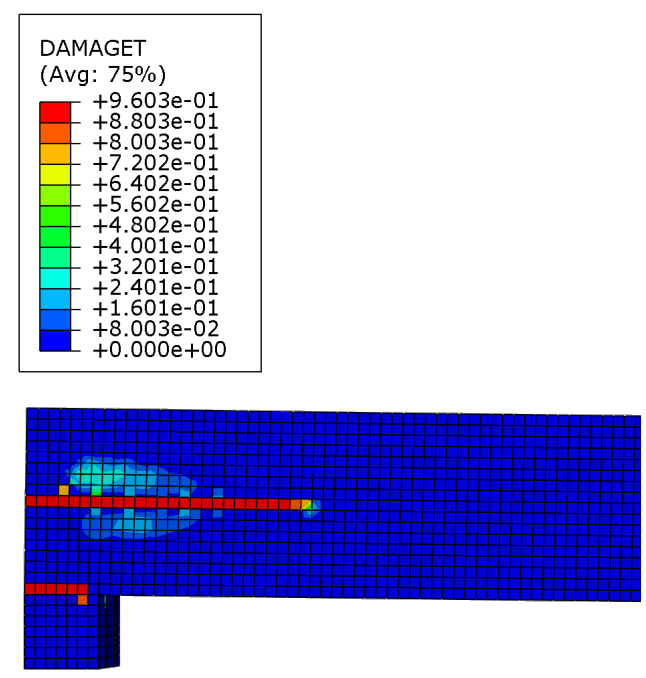

(b)
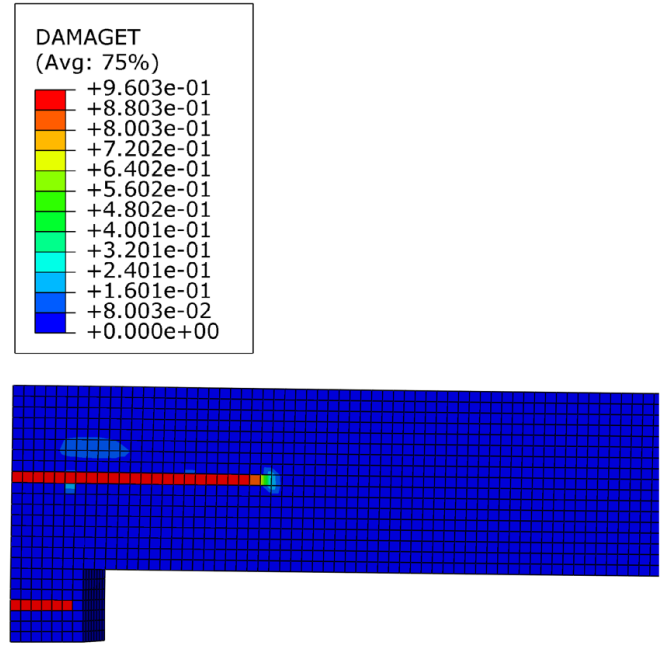

(d)

Figura 10: Dano por tração: modelo VE (a); modelo VEF-3 (b); modelo VEF-4 (c); modelo VEF-5 (d).

Conforme ilustra a Figura 9, entre os modelos investigados, não houve redução significativa do dano à compressão. Tal resultado era esperado, uma vez que a resistência à compressão do concreto não foi alterada e os estribos adicionais não contribuem para o aumento desta propriedade. Contudo, ela teve uma concentração de tensões que foi dispersada com a modelagem da armadura de fretagem.

Por outro lado, no que diz respeito ao dano à tração, as vigas com estribos adicionais (modelos VEF-3, VEF-4 e VEF-5 - Figuras 10b, 10c e 10d, respectivamente), apresentaram uma redução significativa quando comparadas à viga do modelo VE (Figura 10a). Tal fato é corroborado pela menor quantidade de elementos que foram sujeitos à uma falha irreversível durante o processo de fratura. Tal redução fica evidente quando se analisam os valores de tensão nos estribos - vide Figura 11. 


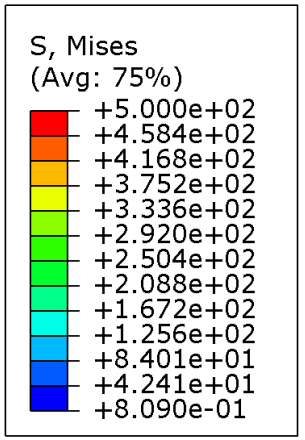

S, Mises (Avg: 75\%)

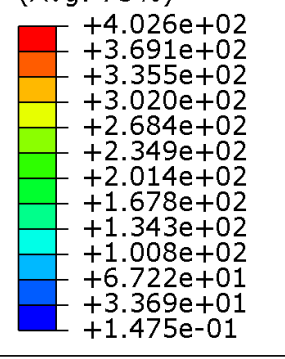

\section{$S$, Mises} (Avg: 75\%)

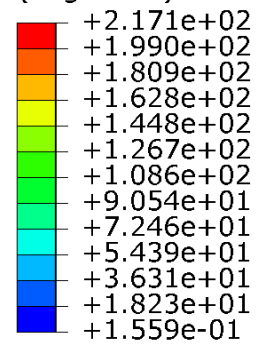

\section{S, Mises}

(Avg: 75\%)

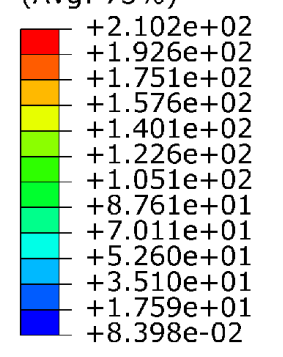

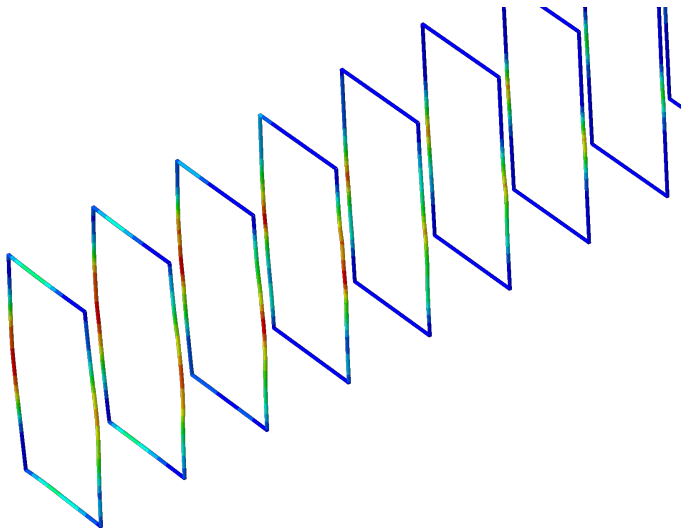

(a)

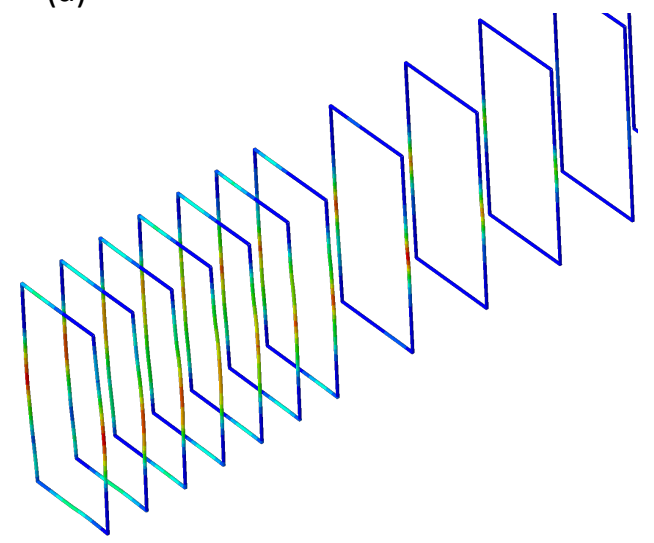

(b)

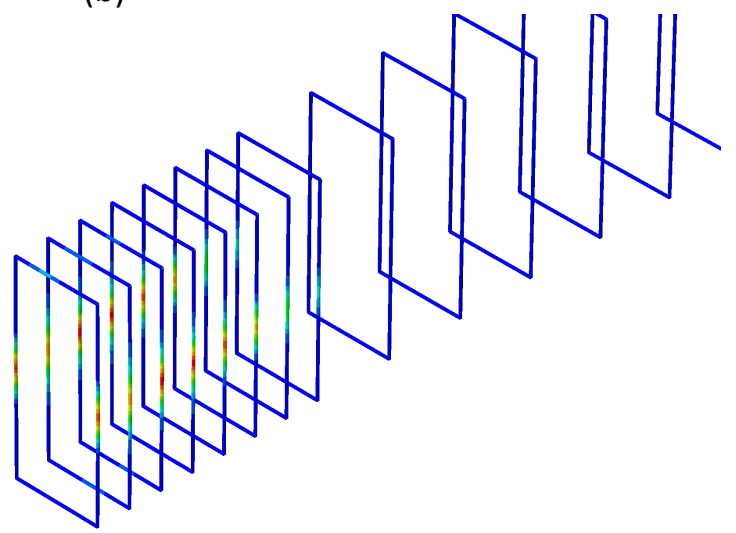

(c)

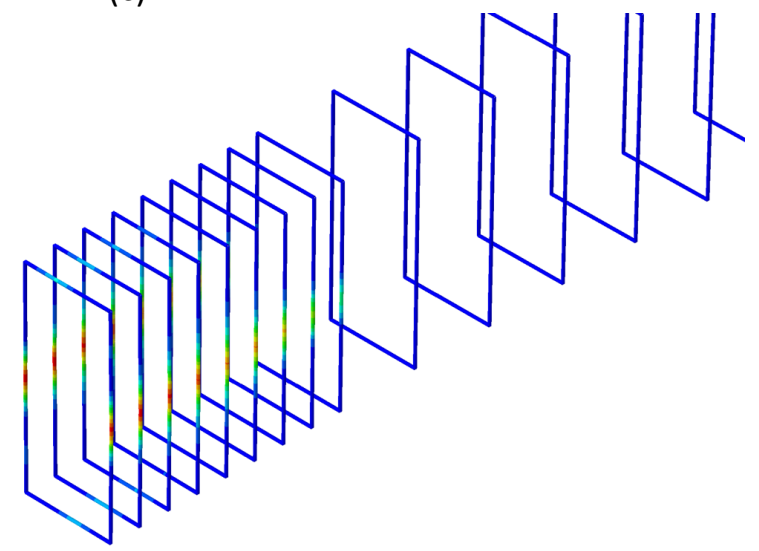

(d)

Figura 11: Valores de tensão nos estribos: modelo VE (a); modelo VEF-3 (b); modelo VEF-4 (c); modelo VEF-5 (d). 
No modelo VE (Figura 11a), a tensão de von Mises atingiu o valor de escoamento do aço (500 MPa), situação indesejada em condições de serviço/utilização, enquanto nos modelos VEF-3, VEF-4 e VEF-5 (Figuras 11b, 11c e 11d, respectivamente), devido à inserção dos estribos adicionais, os valores máximos de tensão foram, de forma respectiva, iguais a 402,6 MPa, 217,1 MPa e 210,2 MPa, ocasionando uma redução de até $57,96 \%$.

\section{CONCLUSÃO}

O presente trabalho contribui para ampliar o conhecimento quanto ao estado de tensões na zona de ancoragem de vigas pós-tracionadas com aderência posterior, apresentando parâmetros que podem ser adotados no dimensionamento de elementos protendidos. Com base na revisão bibliográfica apresentada e nas simulações numéricas, conclui-se que:

- na região das ancoragens, o estado de tensão é complexo, exigindo a adoção de armaduras adicionais para combater os esforços transversais de tração que podem provocar o fendilhamento longitudinal do elemento estrutural;

- a inserção dos estribos adicionais, para combater os esforços transversais de tração, não promoveu alterações na falha à compressão na região das ancoragens, todavia promoveu redução significativa da falha à tração;

- os estribos do modelo VE apresentaram valores de tensão de von Mises superiores aos estribos dos modelos VEF-3, VEF-4 e VEF-5. Os valores de tensões nos estribos do modelo VE (desprovido de estribos adicionais no comprimento de regularização) podem inviabilizar o dimensionamento ao estado limite último deles. Tal fato ressalta a importância da consideração das armaduras de fretagem no dimensionamento de vigas protendidas;

- a utilização de uma quantidade de estribos superior ou inferior à calculada pela Equação (7) não ocasionou alterações significativas no dano à tração nem à compressão no comprimento de regularização. Contudo, a máxima tensão de von Mises nos estribos da ancoragem aumentou, consideravelmente $(85,44 \%)$, ao retirar um estribo e reduziu, de forma pouco expressiva $(3,28 \%)$, ao aumentar um estribo em cada extremidade;

- a Equação (7) mostrou ser uma ferramenta segura para o dimensionamento de armaduras de fretagem, na forma de estribos adicionais, em vigas pós-tracionadas sem aderência. Entretanto, faz-se necessário salientar a necessidade de um maior número de análises e estudos sobre o tema para retificar os métodos de cálculo.

\section{REFERÊNCIAS}

AMERICAN CONCRETE INSTITUTE. (2019). 318-19: Building Code Requirements for Structural Concrete and Commentary.

ASSOCIAÇÃO BRASILEIRA DE NORMAS TÉCNICAS. (2014). NBR 6118: Projeto de estruturas de concreto - Procedimento. Rio de Janeiro. 
AXSON, D. P. (2008). Ultimate Bearing Strenght os Post-tensioned Local Zones in Lightweight Concrete. Thesis (Master of Science In Civil Engineering) - Virginia Polytechinic Institute and State University, Blacksburg VA.

BASE, G. D.; REED, J. B.; BEEBY, A. W.; TAYLOR, H. P. J. (1966). An Investigation of the Crack Control Characteristics of Various Types of Bar in Reinforced Concrete Beams. Research Report № 18, Cement and Concrete Association, London.

BISCHOFF, P. H.; NAITO, C. J.; INGAGLIO, J. P. (2018). Immediate deflection of partially prestressed concrete flexural members. ACI Structural Journal, v. 115, n. 6, p. 1683-9A.

BONETTI, R.; ROBERTS-WOLLMANN, C. L.; SANTOS, J. T. (2014). Bearing Stenght of Confined Concrete. ACI Structural Journal, v. 111, p. 13-17.

BONOPERA, M., CHANG, K. C., CHEN, C. C., SUNG, Y. C., TULLINI, N. (2019). Experimental study on the fundamental frequency of prestressed concrete bridge beams with parabolic unbonded tendons. Journal of Sound and Vibration, v. 455, p. 150-160.

BURDET, O. L. (1990). Analysis and Design of Anchorage Zones in Post-tensioned Concrete Bridges. Dissertation (Doctor of Philosophy) - The University of Texas at Austin, Texas.

CERVENKA, V.; GANZ, H. R. (2014). Validation of Post-tensioning Anchorage Zones By Laboratory Testing and Numerical Simulation. Structural Concrete 15, n. 2.

CHEN, W.; HAO, H.; CHEN, S. (2015). Numerical analysis of prestressed reinforced concrete beam subjected to blast loading. Materials \& Design, v. 65, p. 662-674.

DE OLIVEIRA, D. R. C.; JÚNIOR, O. A. S. (2016). Influência do Traçado do Cabo na Resistência ao Cisalhamento de Vigas de Concreto Protendido. Revista IBRACON de Estruturas e Materiais, v. 9, n. 5.

DU, J. S., AU, F., CHAN, E. K. H., LIU, L. (2016). Deflection of unbonded partially prestressed concrete continuous beams. Engineering Structures, v. 118, p. 89-96.

FUSCO, P. B. (2013). Técnica de Armar Estruturas de Concreto. 2ª Edição, Pini, São Paulo.

GERGELY, P.; SOZEN, M. A. (1967). Design of the Anchorage Zone Reinforcement in Prestressed Concrete Beams. PCI Journal, Vol. 12, p. 63-75.

GUO, Z. (2014). Principles of reinforced concrete. Butterworth-Heinemann.

GUYON, Y. (1953). Prestressed Concrete. John Willy and Sons, New York.

HAROON, S.; YAZDANI, N.; TAWFIQ, K. (2006). Posttensioned Anchorage Zone Enhancement With Fiber-Reinforced Concrete. Journal of Bridge Engineering, p. 566-572.

HENGPRATHANEE, S. (2004). Linear and Nonlinear Finite Element Analyses of Anchorage Zones in Post-tensioned Concrete Structures. Dissertation (Doctor of Philosophy In Civil Engineering) - Virginia Polytechinic Institute and State University, Blacksburg VA. 
HOU, D., ZHAO, J., SHEN, J. S., CHEN, J. (2017). Investigation and improvement of strut-and-tie model for design of end anchorage zone in post-tensioned concrete structure. Construction and Building Materials, v. 136, p. 482-494.

IYENGAR, K. T. S. R.; YOGANANDA, C. V. (1966). A three-dimensional stress distribution problem in the anchorage zone of a post-tensioned concrete beam. Magazine of Concrete Research, $\mathrm{v}$. 18 , n. 55, p. $75-84$.

KIM, J. R.; KWAK, H.; KIM, B.; KWON. Y.; BOUHJITI, E. M. (2018). Finite Element Analyses and Design of Post-tensioned Anchorage Zone in Ultra-high-performance Concrete Beams. Advances in Structural Engineering, p. 1-14.

KOMENDANT, A. E. (1952). Prestressed Concrete Structure. McGraw-Hill, New York.

KWON, Y.; KIM, J.; YANG, J. (2017). Development of efficient anchorage device and estimation of its bearing strength of posttensioning anchorage zone. Journal of Structural Engineering, v. 144 , n. 3, p. 04017219.

MAO, W., GOU, H., YANNIAN H., PU, Q. (2018). Local Stress Behavior of Post-Tensioned Prestressed Anchorage Zones in Continuous Rigid Frame Arch Railway Bridge. Applied Sciences, v. 8, n. 10, p. 1833.

MARTÍ, J. V.; GARCÍA-SEGURA, T.; YEPES, V. (2016). Structural design of precast-prestressed concrete $\mathrm{U}$-beam road bridges based on embodied energy. Journal of Cleaner Production, $\mathrm{v}$. 120 , p. 231-240.

MOREIRA, L. S.; SOUSA JR, J. B. M.; PARENTE JR, E. (2018). Nonlinear finite element simulation of unbonded prestressed concrete beams. Engineering Structures, v. 170, p. 167-177.

MÖRSH, E. (1924). Über die Berechnung der Gelenkquader. Béton und Eisen, № 12.

MOURA, M. W., REAL, M. V., LORIGGIO, D. D. (2018). Momento último de vigas de concreto protendido: validação e cálculo do erro do modelo. Revista IBRACON de Estruturas e Materiais, v. 11, n. 2, p. 307-330.

SHIN, H., YANG, J., YOON, Y., MITCHELL, D. (2016). Mix design of concrete for prestressed concrete sleepers using blast furnace slag and steel fibers. Cement and Concrete Composites, v. 74, p. 39-53.

YETTRAM, A. L.; ROBBINS, K. (1969). Anchorage Zone Stresses in Auxially Post-Tensioned Members of Uniform Rectangular Section. Magazine of Concrete Research, 21, p. 103-112.

YUN, Y. M. (2005). Evaluation of Ultimate Strength of Post-Tensioned Anchorage Zone. Journal of Advanced Concrete Technology, v. 3, n. 1, p. 149-159.

ZHAO, L.; SHEN, S.; WANG, L.; CHEN, J. (2011). A Design Approach for the Interior Anchorage Zone of Post-tensioned Concrete Structure. Journal of Civil Engineering, v. 15, n. 13, p. 487-495. 
ZHI-QI, H.; ZHAO, L. (2011). Investigation of Bursting Forces in Anchorage Zones: CompressionDispersion Models and Unified Design Equation. Journal of Bridge Engineering, November/December, p. 820-827.

ZIELINSKI, J. L.; ROWE, R. E. (1960). An Investigation of the Stress Distribution in the Anchorage Zones of Post-Tensioned Concrete Members. Research Report № 9, Cement and Concrete Association, London. 